\title{
Rehabilitation of Deficient Anterior Maxilla with Three Novel Techniques: A Case Report
}

\author{
Sarika Kapila ${ }^{1}$, Ramandeep S Bhullar ${ }^{2}$, Tejinder Kaur ${ }^{3}$, Harsh D Singh ${ }^{4}$
}

\begin{abstract}
An implant-supported prosthesis offers a more predictable course of treatment as compared to other options available for rehabilitating edentulous arches and dental implants are a more acceptable treatment option to the dental fraternity and the public. Atrophic maxilla can be managed well utilizing autogenous bone grafts from intraoral donor sites, and the primary objective of this case was to use osseodensification as a means of acquiring more bone volume/density. We combined the use of three techniques, i.e., autogenous bone graft from symphysis, osseodensification drilling for implant placement, and Toronto prosthesis for rehabilitation of atrophic maxilla in a 35-year-old male patient. After 1 year of follow-up, the implant-based prosthesis is working well without any complication and established that autograft followed by osseodensification and rehabilitation with Toronto bridge gives good results. Block grafting followed by implant placement with osseodensification technique and Toronto prosthesis yielded great aesthetics and functional results.

Keywords: Atrophic maxilla, Autogenous block graft, Implant-supported prosthesis, Osseodensification.

AMEl's Current Trends in Diagnosis \& Treatment (2021): 10.5005/jp-journals-10055-0119
\end{abstract}

\section{INTRODUCTION}

Long-term edentulism, trauma, ${ }^{1}$ and/or congenitally missing teeth often lack sufficient bone height and width for successful osseointegration of dental implants. ${ }^{2}$ Unfavorable maxillomandibular relationship contributed by an unfavorable pattern of ridge resorption requires angulations of the implants and/or angled abutments and affects the proximity of adjacent facial concavities (maxillary sinus, nasal cavity) and vital structures (mandibular nerve). ${ }^{3}$ In the first year, bone loss after tooth extraction is usually dramatic and irreversible and more prominent. Clinical experience has shown that the minimum required dimensions of bone include a ridge width of $5 \mathrm{~mm}$, allowing for bone on the facial and lingual aspects of the implant, and a vertical bone height of 7-10 mm.

Bränemark et al. in 1975 described the procedure of autologous bone grafting for dental implants that include intraoral sites, like maxillary tuberosity, palate, zygomatic arch, and mandibular coronoid process due to surgical morbidity associated with the extraoral donor sites. ${ }^{4,5}$ Bone obtained from mandibular symphysis and ramus is of intramembranous type, which undergoes less resorption as compared to endochondral bone. An additional advantage of these intraoral sites includes that the harvest can be performed in the same operating field and done on an outpatient basis under regional anesthesia. ${ }^{6}$

Primary and secondary stabilities required for the long-term implant success depend on a number of factors, e.g., implant design (geometrical configuration, implant coating, or porosity), surgical instrumentation (sequence, speed, and instrumentation technique), and patient health status (host response and quality and quantity of bone). Huwais in 2013 developed a new concept of osseodensification drilling and designed specialized burs (Densah $^{\mathrm{TM}}$ burs) for this technique. This technique creates a densified osteotomy site by compaction autografting instead of subtractive bone drilling, which excavates bone during osteotomy preparation. Compaction autografting results in bone preservation and condensations, thus increasing the primary implant stability. ${ }^{7}$

\footnotetext{
${ }^{1-4}$ Department of Oral and Maxillofacial Surgery, Shri Guru Ram Das Institute of Dental Sciences and Research, Amritsar, Punjab, India

Corresponding Author: Sarika Kapila, Department of Oral and Maxillofacial Surgery, Shri Guru Ram Das Institute of Dental Sciences and Research, Amritsar, Punjab, India, Phone: +91 8146536193, e-mail: sarukapila75@gmail.com

How to cite this article: Kapila S, Bhullar RS, Kaur T, et al. Rehabilitation of Deficient Anterior Maxilla with Three Novel Techniques: A Case Report. AMEl's Curr Trends Diagn Treat 2021;5(1):48-52.

Source of support: Nil

Conflict of interest: None
}

Combining advantages of both screw-retained and cementretained prosthesis led to the development of "Toronto prosthesis" described as "abutment-hybrid overdenture" for final rehabilitation. It has implant emergence that can be corrected and a screw hole of each crown that can be fabricated in an esthetically and functionally acceptable area. Drawbacks include complex and expensive fabrication as compared to the conventional prosthesis. ${ }^{8}$

This case report presents the first-ever unique case where three techniques, i.e., autogenous bone augmentation in posttrauma partially atrophic anterior maxilla followed by implant placement using osseodensification technique and prosthetic rehabilitation with Toronto prosthesis, were instituted with a successful outcome.

\section{Case Description}

A 35-year-old male reported to the Department of Oral and Maxillofacial Surgery, Sri Guru Ram Das Dental Science and Research, Amritsar, with a chief complaint of missing upper front tooth for the last 6 months, which got avulsed due to trauma. However, the patient reported no history of any jaw bone fracture. There was no significant history of any chronic systemic disease. 
Teeth numbers $14,13,12,11,21$, and 22 were missing whereas teeth 15,16 , and 18 were grossly carious, and endodontic treatment was advised for tooth 23. A long-span fixed partial denture from tooth number 46 to 37 was used to rehabilitate mandibular missing teeth by his previous dentist. A substantial amount of hard and soft tissue was lost due to trauma. On ridge mapping the width of bone in the region of missing teeth, it was less than $3 \mathrm{~mm}$, which was insufficient for implant placement. A cone-beam computed tomography (CBCT) of the maxilla and mandible was advised to get a precise view. CBCT showed that the amount of alveolar bone width at the edentulous site was insufficient for the successful placement of implant of appropriate dimension (Fig. 1). Evaluation of the transplannar images of mandibular symphysis was done to assess the feasibility of harvesting required bone blocks. Labiolingual thickness and position of the mental nerve were found to be favorable.

Under local anesthesia, a surgical procedure was planned after obtaining a written consent from the patient. As labiopalatal dimensions were insufficient in the maxillary anterior teeth region for implant placement, onlay block grafting was planned (Fig. 2). Mandibular symphysis was selected as a donor site.

\section{Harvesting of Block Graft from Mandibular Symphysis}

Bilateral inferior alveolar nerve blocks (Lignocaine 2\% with adrenaline 1:100,000) were given followed by vestibular incision

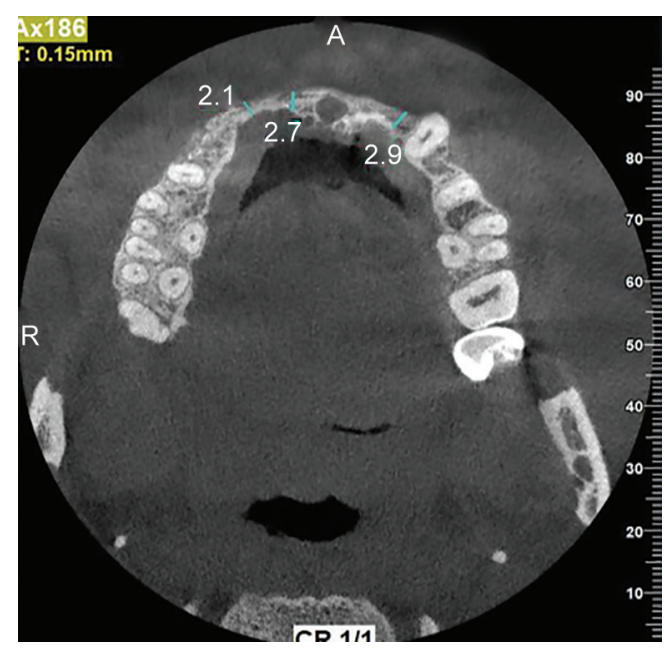

Fig. 1: Preoperative axial section on CBCT. Decreased labiopalatal thickness of the edentulous area was noted

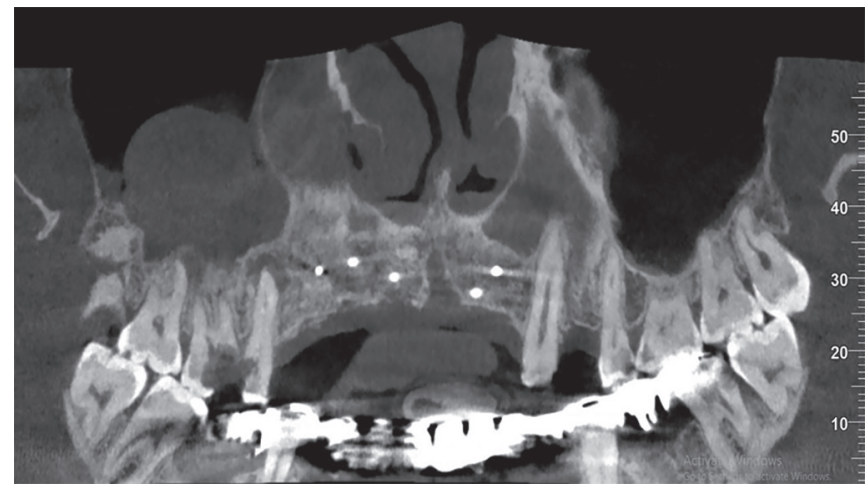

Fig. 2: Postoperative panoramic view-maxillary edentulous ridge, onlay bone graft in situ with titanium screws. Block graft was harvested from the mandibular symphysis region on labial aspect of mandible extending from mesial aspect of right lower premolar to the left one. Two releasing incisions were given to reflect the mucoperiosteal flap. A $10 \times 10 \mathrm{~mm}$ outline was marked on both sides of the symphysis to harvest two separate bone blocks. A piezosurgical unit was then used to harvest the bone block with a minimal thickness of $4 \mathrm{~mm}$ (Fig. 3). The osteotomy site was filled with Osseograft (DMBM-xenograft), and the flap was sutured with 3-0 silk.

\section{Placement of Block Grafts at the Recipient Site}

The recipient site was perforated with fissure bur to induce bleeding and promote revascularization of the graft. Harvested blocks were adapted to the recipient site in region of 11,12 and 21,22 and were held in position with four self-tapping titanium screws (diameter: $2 \mathrm{~mm}$, length: $10 \mathrm{~mm}$ ). Space between the block graft and recipient site was filled with Osseograft (DMBM—xenograft). Absorbable collagen membrane (HEALGUIDE ${ }^{\mathrm{TM}}$ ) was used to cover the area. Interrupted 3-0 vicryl sutures were placed to close the site. Analgesics were prescribed along with postoperative instructions to the patient. Soft tissue healing was uneventful at both sites.

Postoperative 6 months CBCT evaluation showed buccolingual width as ranging from 4.5 to $6.5 \mathrm{~mm}, 3 \mathrm{~mm}$ apical to alveolar crest (Fig. 4). Surgical reentry with implant placement was planned as the postoperative picture after block grafting depicted increased labiopalatal width (Fig. 5).

\section{Implant Placement}

After administering bilateral infraorbital nerve block followed by mucoperiosteal flap elevation (Fig. 6), fixation screws were removed. Implant osteotomy was done with Densah ${ }^{\text {TM }}$ burs (Fig. 7). Three implants of $3.5 \times 11.5 \mathrm{~mm}, 3.5 \times 10 \mathrm{~mm}$, and $3.5 \times 11.5 \mathrm{~mm}$ (Osstem TS-III implants) were placed in regions of 13,11 , and 23 , respectively (Fig. 8). Primary implant stability was measured with MEGA ISQ ${ }^{\text {TM }}$ (Original Ostell Technology) that showed implant stability quotient (ISQ) values as 75, 70, and 73, respectively. After placing cover screws on all three implants, interrupted sutures were placed.

The second-stage implant surgery was done 6 months later after successful confirmation of osseointegration of all three implants as clearly shown in intraoral periapical (IOPA) (Fig. 9) and orthopantomogram (OPG) (Fig. 10). Healing abutments were placed on all three implants after removing cover screws, for a period of 15 days

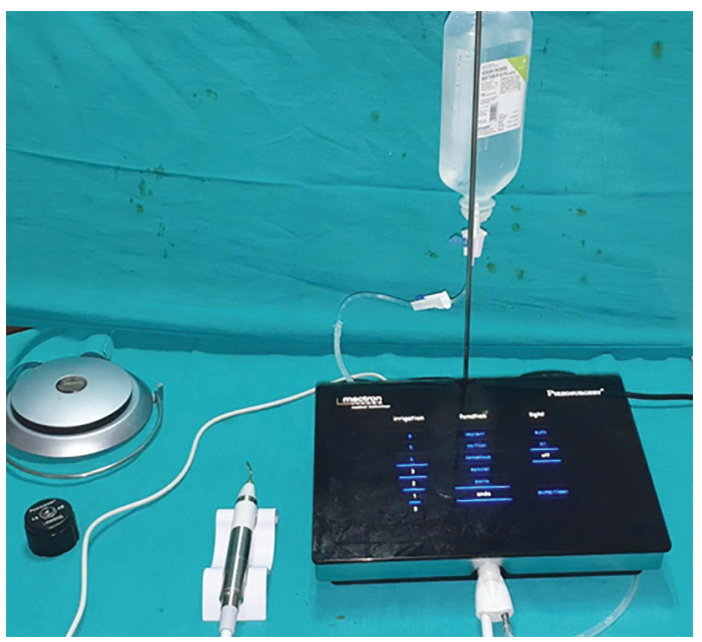

Fig. 3: Piezosurgical unit used for harvesting of symphysis onlay bone graft 


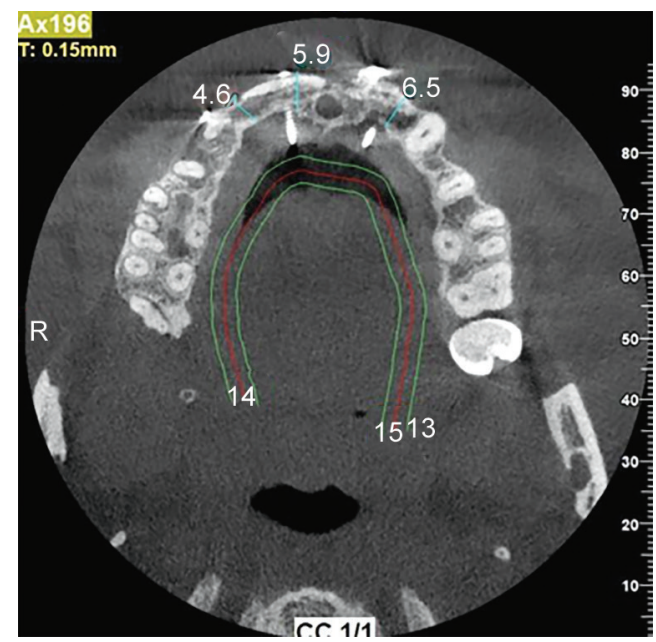

Fig. 4: Postoperative axial section on $C B C T$. Increased labiopalatal thickness of the edentulous area was seen after onlay bone grafting

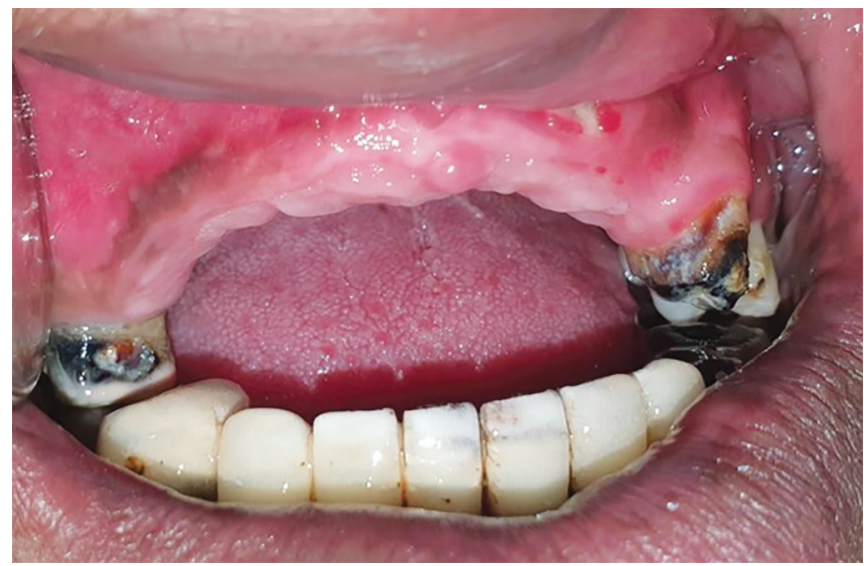

Fig. 5: Postoperative view of the edentulous area showing increased labiopalatal width (6 months postoperative)

(Fig. 11). Maxillary impression was made using the closed technique. A mesostructure with multiple individual abutments was made by casting. After confirming clinically passive fit of structure individual metal-ceramic crowns were made. For replicating soft tissues, the mesostructure was ceramized in pink. The mesostructure was screwed on implants and was given a final torque of $30 \mathrm{~N} \mathrm{~cm}^{-1}$. Metal ceramic crowns were cemented, and excess cement was removed.

The case is being followed for the last 1 year after prosthesis placement, and no complication has been reported till now. To our knowledge and literature search, this is the first reported case with block grafting, osseodensification drilling, and Toronto bridge used in a single patient (Fig. 12).

\section{Discussion}

Maxillary bone resorption in edentulous ridge occurs threedimensionally becoming narrower transversely. ${ }^{9}$ For achieving adequate osteointegration and a predictable implant therapy outcome, the presence of facial-palatal/lingual alveolar width of at least $1-1.5 \mathrm{~mm}$ is necessary. ${ }^{10}$ Guided bone regeneration can be done with resorbable membranes/non-resorbable membrane, crest splitting and ridge expansion, onlay grafting, autogenous bone block graft, and Le Fort I osteotomy with interposition bone grafts.

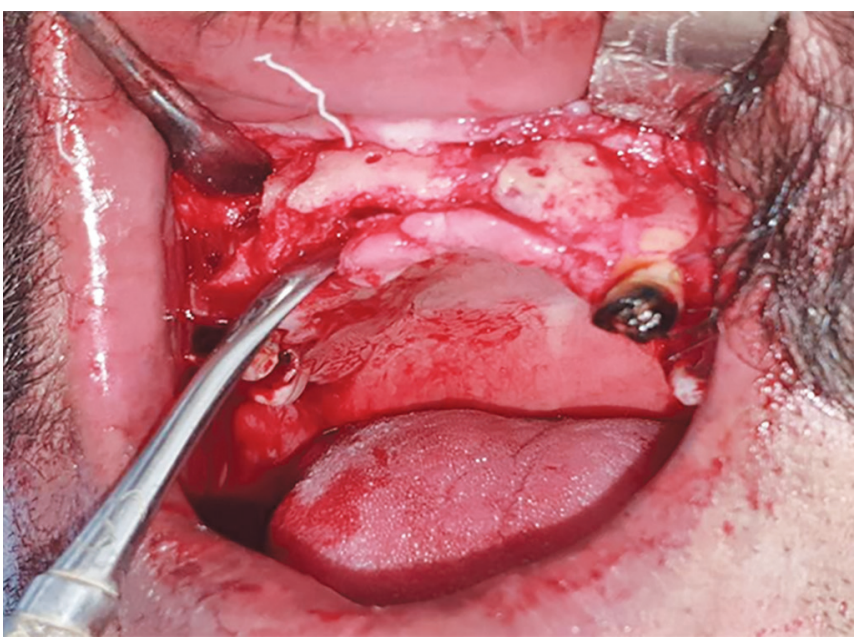

Fig. 6: Exposure of implant site showing increased bone volume

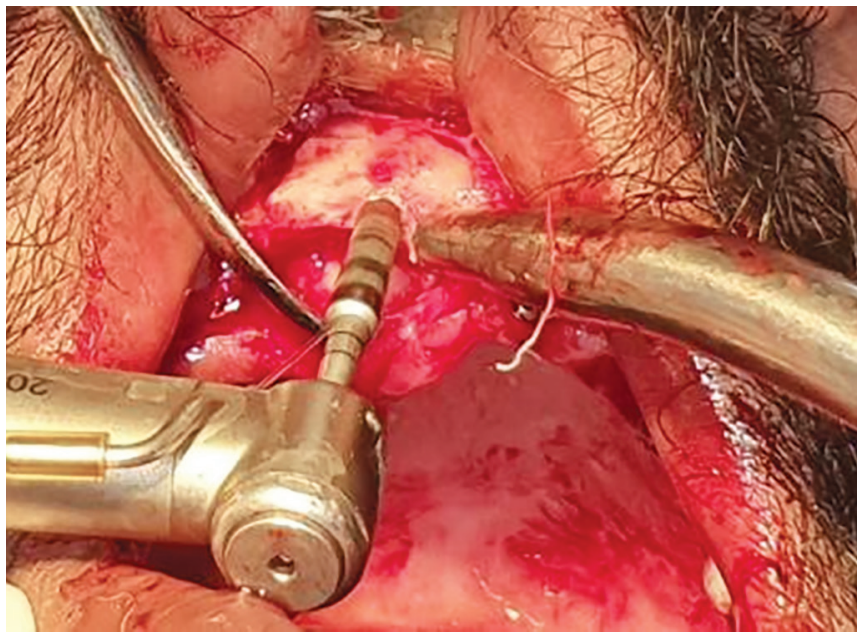

Fig. 7: Implant placement using Densah ${ }^{\mathrm{TM}}$ burs

Based upon computed tomographic evaluation, the average dimensions of bone blocks that can be harvested from the region of mandibular symphysis region are $32.7 \mathrm{~mm}$ in length, $4.87 \mathrm{~mm}$ in width, and $4 \mathrm{~mm}$ in thickness. Though symphysis cannot provide bone for grafting of the entire arch, it can certainly provide adequate bone for grafting of two to six teeth. ${ }^{11}$ Symphysis region certainly provides thicker and larger grafts as compared to mandibular ramus region, resulting in better clinical outcome in alveolar ridge augmentation. In the present case, two bone blocks of dimension $10 \times 10 \times 4 \mathrm{~mm}$ were harvested from the symphysis region, which were adequate for ridge augmentation of anterior maxilla. Additional benefit of using symphysis as a donor site is that it can be reused for second bone block harvesting after a waiting period of 5-6 months between two harvesting procedures from the same area, suggesting that it is a renewable reservoir of high-quality bone. ${ }^{12}$

To overcome the limitation of sensory disturbance in symphysis during autogenous bone block graft using piezoelectric surgery can be used that reduces the risk of damaging surrounding soft tissues and important structures, such as nerves and vessels. ${ }^{13}$ Altiparmak et al. reported temporary paresthesia in $38.5 \%$ of cases in which block was harvested using conventional surgery, while only $3.2 \%$ of cases performed with piezoelectric surgery reported temporary paresthesia. ${ }^{14}$ 


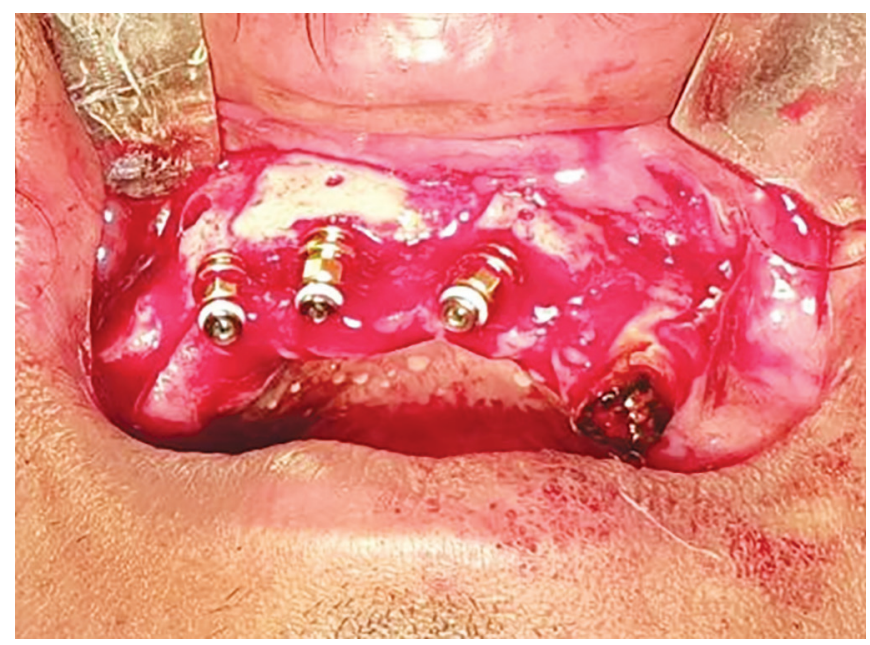

Fig. 8: Implant placed in augmented ridge

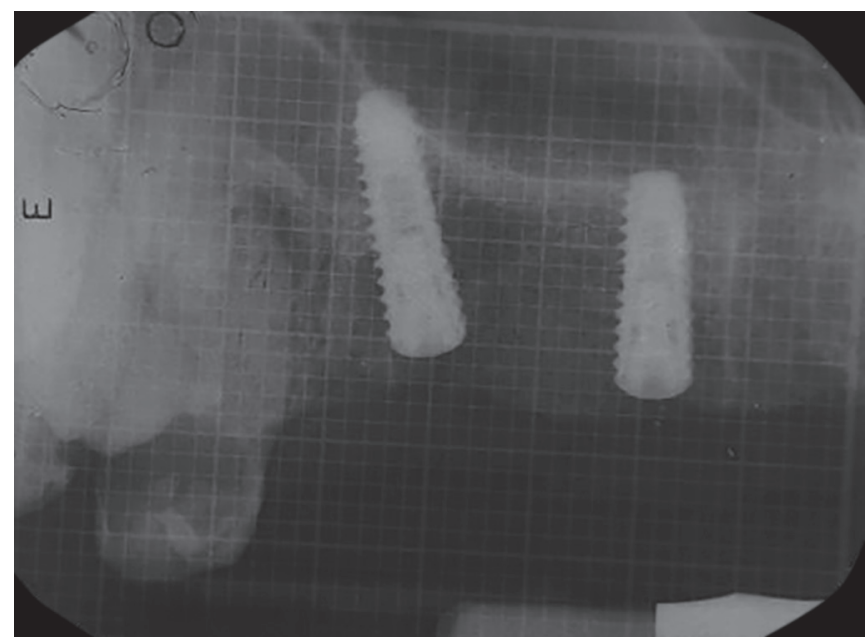

Fig. 9: IOPA of implants placed after 6 months

It is a well-documented fact that autogenous bone blocks exhibit a variable degree of resorption during the healing phase. ${ }^{15}$ Antoun et al. noted that combining symphysis bone graft with membrane limits the resorption rate of harvested graft significantly as compared to cases where the graft is placed alone. Usage of non-resorbable membranes can lead to complications, such as membrane exposure, that can affect regenerative outcomes. ${ }^{16}$ Using a combination of resorbable barrier membranes, such as collagen membranes, along with particulate bone grafts with autogenous bone blocks can limit the resorptive changes. ${ }^{17}$ In the present case, Osseograft (DMBM-xenograft) with collagen membrane was used along with symphysis bone block.

Simultaneous implant placement with ridge augmentation can lead to complications, such as wound dehiscence, infection, and graft failure. This can be readily avoided by delaying implant placement for 4-6 months after autogenous bone block grafting. Insufficient healing time can lead to block bone being separated from implant. ${ }^{18}$ In the present case, implant placement was done after 6 months of ridge augmentation.

In bone deficient regions, standard implant drills can cause buccal and lingual bone dehiscence contributing to low primary stability. ${ }^{19}$ Osseodensification introduced by Huwais $S$ is a bone

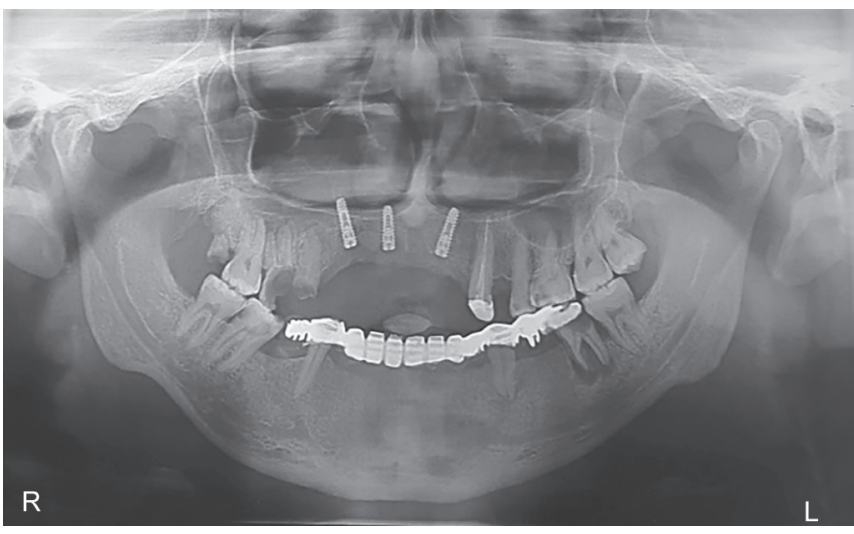

Fig. 10: OPG of implants placed after 6 months

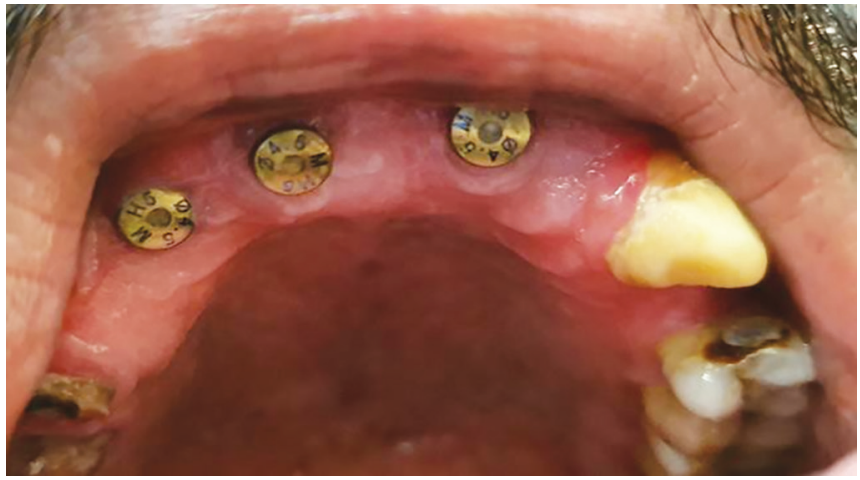

Fig. 11: Healing abutment placed after 6 months of implant placement

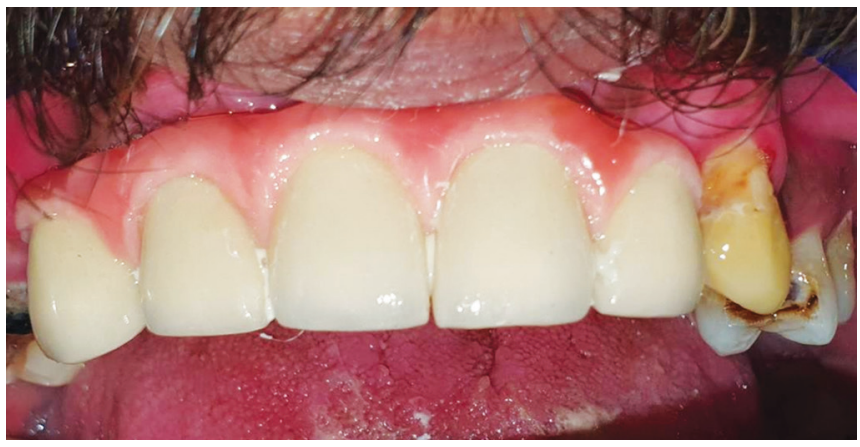

Fig. 12: Toronto prosthesis placement

additive technique, which densifies bone. It preserves bone and condensation during osteotomy preparation, thereby increasing the bone density in peri-implant region. It preserves the bone bulk, increases density, and shortens the healing time.

The specialized drills are used in an anticlockwise direction (densifying mode) with pumping motion (in and out movement) creating dense compact bone along the osteotomy walls. Also, it helps in ridge expansion while maintaining the ridge integrity. Gaikwad et al. in their meta-analysis of animal studies demonstrated that osseodensification significantly improved insertion torque of implants during placement, as it improves the bone density in the prepared osteotomy sites. Also, the histomorphometric analysis showed that it significantly improved bone-implant contact and bone area frequency occupancy due to the presence of autogenous bone fragments in the osseodensified osteotomy sites. ${ }^{20}$ Koutouzis et al. noted that osseodensification caused ridge expansion along 
with higher insertion torque and ISQ in thin ridges. They also recommended that in a situation where alveolar ridge at the crest is only 3-4 mm thick, tissue volume should be developed with guided bone regeneration (GBR) before osseodensification. Hence, in the present case, GBR was performed before osteotomy expansion with osseodensification was done. ${ }^{21}$

In the present case, prosthetic rehabilitation was done with the Toronto bridge technique or abutment-hybrid technique. ${ }^{8,22}$ It was first described by Rajan and Gunaseelan for fabrication of retrievable combined cement-and screw-retained prosthesis for the single-tooth implant-supported prosthesis. ${ }^{23}$ As it is a combination of screw- and cement-retained prosthesis, it allows easy retrievability of abutment and removal of prosthesis for cleaning of excess cement. Toronto prosthesis is indicated for cases demanding esthetics, excessive interocclusal space present for which vertical bone grafting is not possible. ${ }^{24}$ It can be used for restoring single tooth, partial, or completely edentulous arch. It prevents loss of fit of the framework due to refiring and shrinkage caused by porcelain.

Variants available for Toronto prosthesis include the use of casting zirconia, CAD-CAM milled titanium framework and the use of gingiva-colored framework for masking gingival part of the framework. ${ }^{22-24}$

\section{Conclusion}

The inability to restore width and vertical height in deficient maxilla leads to lack of prosthetic rehabilitation in such cases. Block grafting from symphysis followed by implant placement with osseodensification technique and Toronto prosthesis yielded great aesthetics and functional results.

\section{References}

1. Huwais S, Meyer EG. A novel osseous densification approach in implant osteotomy preparation to increase biomechanical primary stability, bone mineral density, and bone-to-implant contact. Int J Oral Maxillofac Implants 2017;32(1):27-36. DOI: 10.11607/jomi.4817.

2. Naruse $\mathrm{Kl}$, Fukuda $\mathrm{M}$, Hasegawa $\mathrm{H}$, et al. Advanced alveolar bone resorption treated with implants, guided bone regeneration, and synthetic grafting: a case report. Implant Dent 2010;19(6):460-467. DOI: 10.1097/ID.0b013e3181fce1a9.

3. Misch $\mathrm{CM}$, Misch CE, Resnik RR, et al. Reconstruction of maxillary alveolar defects with mandibular symphysis grafts for dental implants: a preliminary procedural report. Int J Oral Maxillofac Implants 1992;7(3):360-366. Available at: https://pubmed.ncbi.nlm. nih.gov/1289262/

4. Brånemark $\mathrm{Pl}$, Lindström $\mathrm{J}$, Hallen $\mathrm{O}$, et al. Reconstruction of the defective mandible. Scand J Plast Reconstruct Surg 1975;9(2):116-128. DOI: 10.3109/02844317509022776.

5. Greenberg JA, Wiltz MJ, Kraut RA. Augmentation of the anterior maxilla with intraoral onlay grafts for implant placement. Implant Dent 2012;21(1):21-24. DOI: 10.1097/ID.0b013e3182435ffd.

6. Witek L, Neiva R, Alifarag A, et al. Absence of healing impairment in osteotomies prepared via osseodensification drilling. Int J Periodontics Restorative Dent 2019;39(1):65-71. DOI: 10.11607/ prd.3504.

7. Sultana A, Makkar S, Saxena D, et al. To compare the stability and crestal bone loss of implants placed using osseodensification and traditional drilling protocol: a clinicoradiographical study. J Indian Prosthodont Soc 2020;20(1):45-51. DOI: 10.4103/jips.jips_133_19.
8. Montero J, de Paula CM, Albaladejo A. The "Toronto prosthesis", an appealing method for restoring patients candidates for hybrid overdentures: a case report. J Clin Exp Dent 2012;4(5):e309-e312. DOI: 10.4317/jced.50877.

9. Cawood Jl, Howell RA. Reconstructive preprosthetic surgery: I.Anatomical considerations. Int J Oral Maxillofac Surg 1991;20(2): 75-82. DOI: 10.1016/s0901-5027(05)80711-8.

10. Bassetti MA, Bassetti RG, Bosshardt DD. The alveolar ridge splitting/ expansion technique: a systematic review. Clin Oral Implants Res 2016;27(3):310-324. DOI: 10.1111/clr.12537.

11. Guimarães GM, Bernini GF, Grandizoli DK, et al. Evaluation of bone availability for grafts in different donor areas, through computed tomography. Int J Oral Maxillofac Surg 2019;48:51. DOI: 10.1016/j. ijom.2019.03.155.

12. Schwartz-Arad D, Levin L. Symphysis revisited: clinical and histologic evaluation of newly formed bone and reharvesting potential of previously used symphysial donor sites for onlay bone grafting. J Periodontol 2009;80(5):865-869. DOI: 10.1902/jop.2009.080602.

13. Reininger $D$, Cobo-Vázquez $C$, Monteserín-Matesanz $M$, et al. Complications in the use of the mandibular body, ramus and symphysis as donor sites in bone graft surgery. A systematic review. Med oral Patol Oral Cir Bucal 2016;21(2):e241-e249. DOI: 10.4317/medoral.20938.

14. Altiparmak N, Soydan SS, Uçkan S. The effect of conventional surgery and piezoelectric surgery bone harvesting techniques on the donor site morbidity of the mandibular ramus and symphysis. Int J Oral Maxillofac Surg 2015;44(9):1131-1137. DOI: 10.1016/j.ijom.2015.04.009.

15. Sanz-Sánchez I, Ortiz-Vigón A, Sanz-Martín I, et al. Effectiveness of lateral bone augmentation on the alveolar crest dimension: a systematic review and meta-analysis. J Dent Res 2015;94 (9_suppl):128S-142S. DOI: 10.1177/0022034515594780.

16. Antoun $\mathrm{H}$, Sitbon JM, Martinez $\mathrm{H}$, et al. A prospective randomized study comparing two techniques of bone augmentation: onlay graft alone or associated with a membrane. Clin oral Implants Res 2001;12(6):632-639. DOI: 10.1034/j.1600-0501.2001.120612.x.

17. Mendoza-Azpur G, de la Fuente A, Chavez E, et al. Horizontal ridge augmentation with guided bone regeneration using particulate xenogenic bone substitutes with or without autogenous block grafts: a randomized controlled trial. Clin Implant Dent Relat Res 2019;21(4):521-530. DOI: 10.1111/cid.12740.

18. Kim YK, Ku JK. Ridge augmentation in implant dentistry. J Korean Assoc Oral Maxillofac Surg 2020;46(3):211-217. DOI: 10.5125/ jkaoms.2020.46.3.211.

19. Pai UY, Rodrigues SJ, Talreja KS, et al. Osseodensification-a novel approach in implant dentistry. J Indian Prosthodont Soc. 2018;18(3):196-200. DOI: 10.4103/jips.jips_292_17.

20. Gaikwad AM, Joshi AA, Nadgere JB. Biomechanical and histomorphometric analysis of endosteal implants placed by using the osseodensification technique in animal models: a systematic review and meta-analysis.J J Prosthe Dent 2020. DOI: 10.1016/j.prosdent.2020.07.004.

21. Koutouzis T, Huwais S, Hasan F, et al. Alveolar ridge expansion by osseodensification-mediated plastic deformation and compaction autografting: a multicenter retrospective study. Implant Dent 2019;28(4):349-355. DOI: 10.1097/ID.0000000000000898.

22. Uludag B, Ozturk O, Celik G, et al. Fabrication of a retrievable cement-and screw-retained implant-supported zirconium fixed partial denture: a case report. J Oral Implantol 2008;34(1):59-62. DOI: 10.1563/1548-1336(2008)34[59:FOARCA]2.0.CO;2.

23. Rajan M, Gunaseelan R. Fabrication of a cement-and screw-retained implant prosthesis. J Prosthe Dent 2004;92(6):578-580. DOI: 10.1016/j. prosdent.2004.09.009.

24. Cicciù $M$, Risitano $G$, Maiorana $C$, et al. Parametric analysis of the strength in the "Toronto" osseous-prosthesis system. Minerva Stomatol 2009;58(1-2):9-23. Available at: https://pubmed.ncbi.nlm. nih.gov/19234433/ 